\title{
Esfuerzos realizados en Puerto Rico hacia la consolidación de políticas públicas para la prevención de cánceres asociados al VPH
}

\author{
Diana T Medina-Laabes, ${ }^{1}$ Vivian Colón-López, ${ }^{1}$ Vilnery Rivera-Figueroa, ${ }^{1}$ Coralia Vázquez-Otero, ${ }^{2}$ \\ Glizette O Arroyo-Morales, ${ }^{1}$ Lara Arce-Cintrón, ${ }^{3}$ Paola Fernández-Rivera, ${ }^{4}$ Idamaris Vega, ${ }^{5}$ \\ Roxana Soto-Abreu, ${ }^{1}$ Olga L Díaz-Miranda, ${ }^{1}$ Ángel Rivera, ${ }^{6}$ Iris Cardona, ${ }^{6}$ Ana P. Ortiz, ${ }^{1}$ \\ Lilliam Rodríguez Capó, ${ }^{7}$ y Pamela C. Hull ${ }^{8}$
}

Forma de citar

\begin{abstract}
Medina-Laabes DT, Colón-López V, Rivera-Figueroa V, Vázquez-Otero C, Arroyo-Morales GO, Lara Arce-Cintrón L et al. Esfuerzos realizados en Puerto Rico hacia la consolidación de políticas públicas para la prevención de cánceres asociados al VPH. Rev Panam Salud Publica. 2022;46:e3. https://doi.org/10.26633/RPSP.2022.3
\end{abstract}

RESUMEN

El propósito de este informe especial es describir cronológicamente los eventos que contribuyeron al desarrollo y aprobación de la legislación e implementación del requisito escolar de vacunación en Puerto Rico (PR), con el fin de prevenir el VPH y los cánceres asociados a este. A partir del 2010, PR inició las aprobaciones de políticas públicas con el objetivo de mejorar el registro de casos de los cánceres y la cobertura de la vacuna contra el VPH a través de los planes médicos en adolescentes de 11 a 18 años. En el 2014, los esfuerzos científicos y comunitarios lograron documentar la magnitud de las enfermedades causadas por el VPH, y desarrollar en conjunto, estrategias de prevención y promoción de la vacuna contra el VPH. En agosto de 2018, PR logró ser uno de los primeros cuatro territorios de los Estados Unidos de América en implementar la vacuna contra el VPH como requisito escolar con el fin de disminuir la incidencia de cánceres asociados al VPH en la isla. En el 2019 se garantizó por ley que todo proveedor de vacunación debe reportar al Registro de Inmunización. El caso de PR demuestra que el desarrollo de políticas públicas junto con colaboraciones entre coaliciones académicas, científicas y comunitarias, logran cambios poblacionales y resultados medibles dirigidos a la prevención de VPH. Países con una problemática de salud pública similar podrían adoptar esfuerzos similares a los presentados, y alinearlos al objetivo de la Organización Mundial de la Salud: erradicación del cáncer cervical para 2030.

Palabras claves Vacunas contra papillomavirus; infecciones por papillomavirus; prevención de enfermedades; política pública; Puerto Rico.

\footnotetext{
1 División de Ciencias de la Población y Control del Cáncer de Puerto Rico, Centro Comprensivo de Cáncer de la Universidad de Puerto Rico, San Juan, Puerto Rico. $\triangle$ Vivian Colón-López, vivian.colon@upr.edu

2 Department of Public Health. College for Health, Community and Policy, University of Texas at San Antonio, San Antonio, Texas, Estados Unidos de América.

3 Baylor University, Robins College of Arts and Sciences, Waco, Estados Unidos de América.

4 Facultad de Ciencias Naturales, Recinto de Río Piedras, Universidad de Puerto Rico, San Juan, Puerto Rico.
}

\footnotetext{
5 Departamento de Bioestadística y Epidemiología, Escuela Graduada de Salud Pública, Recinto de Ciencias Médicas, Universidad de Puerto Rico, San Juan, Puerto Rico.

6 Programa de Inmunización, Departamento de Salud de Puerto Rico, San Juan, Puerto Rico.

7 VOCES PR.org; Coalición de Inmunización y Promoción de la Salud de Puerto Rico, Guaynabo, Puerto Rico.

8 Department of Behavioral Science, University of Kentucky, College of Medicine, Markey Cancer Center, 1100 Veteran Drive, Medical Behavioral Science Building, Lexington, Estados Unidos de América.
} 
El impacto a largo plazo de la persistencia del virus de papiloma humano (VPH) en Puerto Rico (PR), ha sido documentado previamente con las altas tasas de incidencia de cáncer cervical y otros cánceres causados por VPH $(1,2)$. Desde 1951, y bajo la Ley Núm. 28 del 20 de marzo, Puerto Rico cuenta con un sistema para el registro de casos de cáncer en la isla, siendo el pionero en establecer este tipo de registro en Latinoamérica $(3,4)$. En la década de 1990, el registro perdió fondos y por ende recursos (3), limitando la cantidad de los datos y su calidad. Sin embargo, en los primeros años del siglo XXI se retomó la importancia de tener un registro de cáncer para la planificación y evaluación de los programas de tratamientos y prevención. La Ley Núm. 28, luego enmendada por la Ley Núm. 113 del 30 de julio de 2010, tiene como objetivo mejorar la calidad y el tiempo del reporte de casos a través de un reporte electrónico (3). Desde el 2017, el Registro Central de Cáncer de Puerto Rico, ha sido reconocido con la calidad clasificada de oro 'Gold Standard for Registry Certification', según la Asociación Norteamericana de Registros Centrales de Cáncer. Esto ha permitido documentar la epidemiología de estas malignidades asociadas al VPH, incluyendo el cáncer cervical, el cual es de los más frecuentemente diagnosticados en la isla $(1,2,4,5)$. Por otro lado, la vacuna contra el VPH para la prevención de cánceres asociados a este virus trajo consigo una serie de esfuerzos a nivel comunitario, de gobierno, de políticas públicas y a nivel científico. El propósito de este informe especial es describir cronológicamente los eventos que contribuyeron al desarrollo y aprobación de la legislación, y la implementación del requisito escolar de vacunación en PR, con el fin de prevenir el VPH y sus cánceres asociados, también serán presentados los eventos ocurridos durante los pasados 15 años que influenciaron positiva o negativamente el desarrollo del requisito escolar de vacunación en PR (figura 1). Finalmente, se discuten las lecciones aprendidas y recomendaciones que podrían informar a otros países la adaptación de políticas públicas para la reducción de cánceres asociados al VPH.

\section{MAGNITUD DE CÁNCERES ASOCIADOS AL VPH EN PUERTO RICO: LA IMPORTANCIA DEL REGISTRO CENTRAL DE CÁNCER}

Un estudio con datos del Registro Central de Cáncer de Puerto Rico entre 2001 al 2017, indicó que las mujeres nacidas en 1991 en la isla, tienen cuatro veces mayor riesgo de ser diagnosticadas con cáncer cervical en comparación con aquellas nacidas en 1961, posiblemente por mayor exposición al VPH (IRR:4.3 [95\% CI, 1.9-9.5]) (6). Puerto Rico, presentó la incidencia más alta de cáncer cervical (11,7 por cada 100000 habitantes) en comparación con otros estados y territorios de los Estados Unidos de América (EU) para el periodo 2008-2012 (7). Datos más recientes indican que entre los cánceres asociados al VPH, el cáncer de orofaringe fue el $5^{\text {to }}$ cáncer más diagnosticado entre los hombres $(4,0 \%)$, en comparación con el total de cánceres diagnosticados (2). Además, según registros obtenidos desde el año 2000 al 2016, mostraron un total de 991 casos de cáncer anal, lo que representa un incremento de casos de carcinoma de células escamosas del ano en un 4,90\% anual (8). Estos datos demuestran la magnitud de los cánceres asociados a VPH, y la necesidad de un plan de acción basado en evidencia científica para reducir este impacto.

Desde el año 2004, varios estudios epidemiológicos se han llevado a cabo con el fin de estimar la magnitud de la infección por VPH en distintas áreas anatómicas (boca, cérvix, pene, ano), $\mathrm{y}$ en diversas poblaciones (mujeres, hombres que tienen sexo con hombres y personas que viven con VIH). Estos estudios han reportado estimados de prevalencia de VPH desde un 21\% hasta un 79\% (9-12). Sin embargo, la mayoría de estos estudios han sido realizados en escenarios clínicos, por lo que la carga de la infección por VPH a nivel poblacional aún se desconoce.

\section{APROBACIÓN DE LA VACUNA CONTRA EL VPH: 2006-2012}

En el 2006, la Administración de Drogas y Alimentos (FDA, por sus siglas en inglés) de los Estados Unidos (EU) aprobó la primera vacuna contra el VPH en el mercado, Gardasil @ tetravalente (contra VPH tipo: 16, 18, 6 y 11) para féminas de 9 a 26 años. De forma continua, el Comité Asesor sobre Prácticas de Inmunización (ACIP, por sus siglas en inglés) de EU recomendó como parte de la rutina de vacunación, la serie de tres dosis de la vacuna contra VPH para niñas de 11 a 12 años. Esto debido a la fuerte respuesta de anticuerpos que se producen luego de la vacunación en esas edades (13).

En el 2009, la FDA aprobó una segunda vacuna, Cervarix® bivalente (contra VPH tipo: 16 y 18) para féminas de 10 a 25 años. En ese mismo año, FDA aprobó el uso de la vacuna Gardasil ${ }^{\circledR}$ tetravalente en varones de 9 a 26 años para prevención de verrugas. Aunque en el 2009 ACIP incluyó unas guías para el uso de esta vacuna en varones, no fue hasta el 2011 que recomienda la vacuna contra el VPH en varones de 11 a 12 años e incluyendo la prevención a cánceres relacionados a VPH (tales como cáncer de año y orofaringe) (13).

\section{POLÍTICA PÚBLICA EN PUERTO RICO EN FAVOR DE LA VACUNA CONTRA EL VPH: 2010-2012}

Datos epidemiológicos sobre el cáncer cervical en PR y demás cánceres asociados al VPH, dieron paso a la aprobación de la Ley Núm. 9 del 20 de enero de 2010. Esta ley garantiza que la vacuna contra el VPH sea cubierta de manera gratuita por cualquier plan médico (público o privado) u organización de servicios de salud para todas las féminas de 11 a 18 años para la prevención de cáncer cervical. Esta ley fue luego enmendada (Ley Núm. 255 de 15 de septiembre de 2012) para incluir en la cobertura a los varones entre 11 a 18 años (14). Siendo Puerto Rico un país donde sobre el $40 \%$ de las familias viven en un nivel de pobreza (15), esta legislación significó un gran paso en la accesibilidad de la inmunización contra el VPH y la salud pública.

\section{NUEVA GENERACIÓN DE LA VACUNA CONTRA EL VPH: $2014-2016$}

En diciembre del 2014, la FDA aprobó la nueva generación de la vacuna Gardasil 9 (nonavalente), la cual expandió su cobertura para incluir 5 tipos adicionales de VPH oncogénicos, no incluidos previamente en la vacuna tetravalente (VPH tipos: 31, $33,45,52,58)(16)$. ACIP recomendó la vacuna nonavalente continuando con la serie de tres dosis en el 2015. En el 2016, luego de examinar los resultados de ensayos clínicos que demostraron la efectividad con solo dos dosis de la vacuna nonavalente en menores de 15 años, hicieron un cambio en la serie de vacunación. Solo se administrarían 2 dosis a menores de 15 años que iniciaran la serie de vacunación contra VPH. Mientras que 
FIGURA 1. Línea de tiempo sobre esfuerzos dirigidos hacia una política pública para la prevención de cánceres asociados al virus de papiloma humano en Puerto Rico.

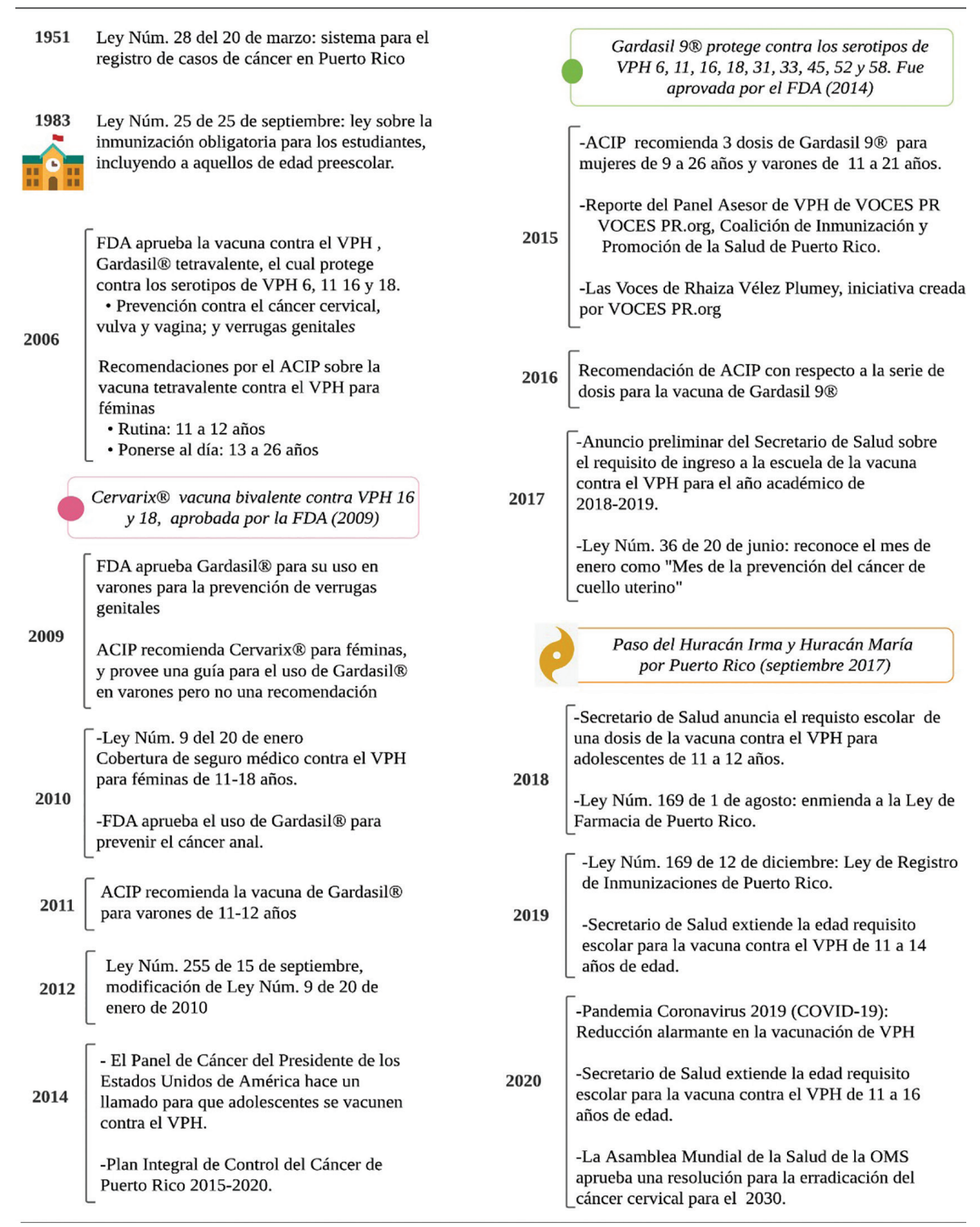

Fuente: elaborado por los autores

VPH: virus de papiloma humano; FDA: siglas en inglés de la Administración de Drogas y Alimentos; ACIP: siglas en inglés de Comité Asesor sobre Prácticas de Inmunización; OMS: Organización Mundial de la Salud

aquellos que iniciarán la vacunación a partir de los 15 años en adelante, continuarían recibiendo la serie de 3 dosis.(17).

\section{PANEL DE CÁNCER DEL PRESIDENTE DE LOS ESTADOS UNIDOS Y LOS ESFUERZOS PARALELOS EN PUERTO RICO: 2014-2015}

El Panel de Cáncer del Presidente de Estados Unidos fue establecido por ley en 1971, y tiene como misión monitorear las actividades del Programa Nacional de Cáncer y reportar al presidente de los EU sobre las barreras para el progreso en la reducción de la carga del cáncer. A principios del 2014, el Panel de Cáncer del Presidente de Estados Unidos emitió un reporte enfatizando la prioridad de la vacunación contra VPH en la agenda de salud pública del país para la posibilidad de un futuro sin cánceres relacionados al VPH (18). Los puntos de acción presentados por el Panel fueron los siguientes: 1) aumentar la vacunación de VPH, debido a la baja tasa de vacunación (menos del 35\% habían completado la serie en los EU); 2) reducir las oportunidades pérdidas en clínicas, promoviendo la comunicación entre profesionales de la salud y los padres; 3) incrementar la aceptación de la vacuna contra el VPH; y 4) maximizar el acceso a la vacuna contra el VPH.

Durante el mismo periodo, se publicó el Plan Integral de Cáncer de Puerto Rico 2015-2020. Este plan describe como organizaciones y coaliciones desarrollarían estrategias específicas de salud pública en el área de cáncer, estableciendo unos objetivos específicos con la expectativa de cumplirlos en 5 años. El primer objetivo de este plan fue aumentar el porcentaje de 
la serie de vacunación completa contra VPH de $17 \%$ a $80 \%$ en jóvenes entre 13 a 17 años para el 2020 (19).

Las estrategias para alcanzar estas metas fueron las siguientes: 1) educar a los proveedores para que recomienden enfáticamente la vacunación contra el VPH a padres y jóvenes; 2) colaborar con organizaciones comunitarias para llevar a cabo actividades educativas y de divulgación, con el fin de aumentar la conciencia pública sobre los beneficios de la vacuna contra el $\mathrm{VPH}$; 3) promover actividades educativas en la escuela para aumentar el conocimiento de los beneficios de la vacuna contra el VPH entre los estudiantes elegibles por edad; 4) apoyar las clínicas escolares que ofrecen la vacuna contra el VPH a bajo costo o sin costo para los estudiantes; 5) integrar a líderes jóvenes para brindar educación sobre las vacunas contra el VPH; 6) apoyar campañas de promoción de la salud dirigidas a padres e individuos elegibles por edad para aumentar su conocimiento de los beneficios de la vacuna contra el VPH, incluida la prevención del cáncer; 7) promover la implementación de los sistemas de recordatorios en el consultorio para aumentar el número de pacientes elegibles por edad que inician y completan la serie de vacunación contra el VPH; y 8) promover el uso del registro de vacunas de Puerto Rico entre los proveedores.

En el 2015, la coalición de vacunación, VOCESPR.org bajo su Panel Asesor de VPH, compuesto de expertos e investigadores en el tema, hizo un reporte para el Gobierno de Puerto Rico estableciendo la necesidad de educación sobre VPH en diferentes sectores ( médicos, maestros, organizaciones religiosas, prensa), e incrementar la vacunación contra el VPH según las recomendaciones de ACIP (20). Entre las estrategias propuestas estuvieron: educar en el tópico a padres, jóvenes y a los sectores que influyen en la decisión sobre la vacunación contra el VPH; además del apoyo educativo proveer apoyo financiero relacionado a la vacunación contra VPH a jóvenes de 11 a 18 años de edad; establecer al Registro de Inmunización de PR como un registro central y robusto sobre la vacunación en la isla; e implementar la vacuna contra el VPH como un requisito escolar para la entrada a escuelas públicas y privadas. Al comparar los puntos de interés del Panel de Cáncer del Presidente y el Panel Asesor de VPH de la coalición de VOCES PR, se observan diferencias entre las necesidades y estrategias mencionadas. Se notan puntos más específicos en la estrategia de PR tales como: educar los sectores influyentes, apoyo económico, trabajar con el registro, y establecer la vacuna contra VPH como requisito escolar.

\section{ACCIÓN PARA LA PREVENCIÓN DE VPH: ESFUERZOS COMUNITARIOS EN PUERTO RICO (2015-2017)}

En 2015, se creó la iniciativa Las Voces de Rhaiza Vélez Plumey con la misión de educar sobre la prevención del cáncer de cuello uterino. Esta iniciativa es basada en el caso de una joven madre de 32 años que murió a causa del cáncer cervical. En sus últimas semanas de vida, Vélez Plumey trabajó activamente en concientizar a las jóvenes a realizar las pruebas de Papanicolaou de manera oportuna (21). A través de las plataformas sociales, esta iniciativa tiene alrededor de 23 mil seguidores (22). Por otra parte, el mes de enero se reconoció como el mes de la prevención de cáncer de cuello uterino bajo la Ley. Núm. 36 del 20 de junio de 2017. De acuerdo con la exposición de esta ley, este mes sería enfocado en la promoción y desarrollo de diversas campañas educativas enfocadas en la prevención de la infección de VPH para los puertorriqueños (14).

\section{LEY DE INMUNIZACIÓN COMPULSORIA}

La Ley Núm. 25 del 25 de septiembre de 1983, según enmendada, se conoce como la ley de inmunización compulsoria para niños de pre-escolar y estudiantes de PR. Esta ley establece unas vacunas obligatorias para todo niño-preescolar y estudiante que vayan a centros educativos (públicos o privados) (23). Esta misma ley le otorga al secretario de salud de PR la autoridad de añadir o eliminar vacunas dependiendo de la necesidad en salud. Además, la ley indica que el secretario de salud deberá anunciar tres meses antes de inicio de clase, los requisitos de vacunación para el nuevo año escolar.

En junio de 2017, el secretario de salud anunció que la vacuna contra el VPH sería requisito para el próximo año escolar (20182019) para jóvenes en el rango de edad de 11 a 12 años (20). Factores como el caso de Rhaiza Vélez Plumey, el informe del Panel Asesor de VPH de VOCES PR.org, los datos de registro de cáncer y estudios epidemiológicos, crearon una corriente de interés socio-político que añadido al poder que otorga la Ley 25 al secretario de salud sobre la inclusión de vacuna para requisito escolar, hizo viable esta intervención de base poblacional (20). Por otra parte, esta decisión del secretario de salud promovió el desarrollo y presentación de varios proyectos en las asambleas legislativas relacionados a la prevención de VPH (cuadro 1).

CUADRO 1. Piezas legislativas presentadas entre los años 2018 y 2019 con respecto al proceso de vacunación en Puerto Rico.

Pieza Legislativa

Proyecto de la Cámara 1576 (2 de mayo de 2018)

Ley de Farmacia de PR, Ley Núm. 169 del 1 de agosto de 2018

Ley. Núm 169 del 12 de diciembre de 2019

\section{Hechos y descripción}

Hecho: las exenciones de vacunación por razón religiosa y médica son permitidas en PR. Descripción: este proyecto proponía incluir la exención filosóficas para que los padres optaran por no vacunar a sus hijos con esta vacuna por razones personales sin base religiosa o médica (32). Sin embargo, no fue aprobado.

Hecho: Ios farmacéuticos solo podían vacunar a personas mayores de 18 años de edad. Descripción: se hace una enmienda, facultando a los farmacéuticos certificados a administrar vacunas a personas de 12 años o más (14).

Hecho: el Registro de Inmunización de PR se creó a través de una orden administrativa en 1994, pero carecía de ciertos poderes legales para lograr agilizar la calidad y obtención de los datos. Descripción: Con el propósito de robustecer el sistema del Registro de Inmunización de PR (PRIR, por sus siglas en inglés), en el cual se ordena a todo proveedor de vacunación reportar al registro la información de cada paciente inmunizado e información adicional (14). 


\section{EFECTOS DE LOS HURACANES IRMA Y MARÍA EN LAS CLÍNICAS DE VACUNACIÓN EN PUERTO RICO}

En septiembre de 2017, PR se vio afectado por dos huracanes de gran fuerza, los huracanes Irma y María, los cuales ocasionaron miles de muertes, y pérdidas millonarias a la infraestructura del país, incluyendo edificios de agencias gubernamentales, hospitales, centros de cuido y clínicas de salud primaria. Las clínicas del Programa Federal Vacunas para Niños (Vaccine for Children Program, en inglés) cuyo propósito es brindar vacunas a niños elegibles, fueron afectadas. A 10 días del evento, solo un $5 \%$ estaban en función (24).

\section{RENUENCIA A LA VACUNACIÓN CONTRA VPH EN PUERTO RICO}

La renuencia a las vacunas es otro continuo reto los programas de inmunizaciones. De acuerdo con la Organización Mundial de la Salud (OMS), la renuencia ante la vacuna se define como el retraso en la aceptación o el rechazo de la vacunación a pesar de la disponibilidad de servicios de vacunación, y se consideró una de las 10 amenazas a la salud en el 2019 (25). En los resultados preliminares de entrevistas realizadas a padres del área metropolitana de PR entre marzo a noviembre 2019 sobre su percepción acerca de la vacuna contra VPH como requisito escolar, se observó que las barreras principales identificadas fueron el poco conocimiento o comprensión sobre la vacuna de VPH y la preocupación sobre los efectos adversos. Además, los mismos padres expresaron que las escuelas y los proveedores de salud deberían de involucrarse en la orientación sobre esta y otras vacunas requeridas para asistir a los centros educativos (26). En otro estudio cualitativo, datos preliminares de entrevistas a informantes claves que están en oposición a la vacuna contra el VPH y al requisito escolar expresaron que su principal motivo de renuencia es la interferencia excesiva del gobierno para tomar decisiones por ellos y violar sus derechos como ciudadanos, como padres y el derecho a la educación (27).

\section{LA PANDEMIA CORONAVIRUS (COVID-19) Y CAMBIOS EN EL REQUISITO DE VACUNACIÓN}

En marzo del 2020, la OMS declaró un estado de pandemia por el nuevo coronavirus, SARS-COV-2, causante de la enfermedad llamada COVID-19 (28). Debido a este evento hubo una interrupción de la cadena de distribución para la vacuna contra VPH, por lo que se estimó una reducción de más del $70 \%$ en la solicitud de compra de esta vacuna en el 2020 en los EU (29).

\section{IMPACTO DE LA POLÍTICA PÚBLICA DE REQUISITO ESCOLAR EN VACUNACIÓN VPH}

La vacuna contra el VPH se inició como requisito escolar en PR en agosto de 2018 para niños y niñas de 11 a 12 años de edad. Con el propósito de tener un mayor alcance en la población de jóvenes, este requisito fue extendiendo su rango de edad, hasta llegar a los 16 años de edad (cuadro 2).

Por otro lado, a pesar de los eventos climáticos, renuencia hacia la vacuna y potenciales barreras socio políticas antes y durante la implementación de este requisito escolar, se observó un potencial aumento en la tasa de vacunación contra VPH. Un análisis preliminar utilizando los datos del Registro de Inmunización de Puerto Rico del 2017 y 2019, se observó un aumento de $54,0 \%$ en la tasa de iniciación de esta vacuna entre jóvenes de 11 a 12 años de edad (30). No obstante, el efecto de la pandemia en las tasas de iniciación y cumplimiento de la serie de vacunación contra VPH aún se desconoce.

\section{UN FUTURO SIN CÁNCER CERVICAL}

La Asamblea Mundial de Salud de la OMS oficializó en noviembre de 2020 el proceso para la erradicación del cáncer cervical para el 2030 a nivel mundial. La meta es alcanzar un $90 \%$ de vacunación contra $\mathrm{VPH}$, un $70 \%$ en prueba de tamizajes y un $90 \%$ a tratamiento de lesiones precancerosa y cáncer asociado a VPH (31).

En este informe especial, describimos de forma cronológica los eventos que contribuyeron al desarrollo e implementación del requisito escolar de vacunación en PR; con el fin de prevenir el VPH y sus cánceres asociados. La contribución conjunta de estos eventos como el reporte del Panel De Cáncer del Presidente, el informe del Panel Asesor de VPH de VOCES PR, el caso de Rhaiza Vélez Plumey y el movimiento de campañas educativas, y la data de estudios epidemiológicos y el Registro Central de Cáncer creó una corriente de interés público y político que llevó a la implementación de la vacuna del VPH como requisito escolar en PR. Sin embargo, los huracanes en el 2017 demostraron unas limitaciones de infraestructura y respuesta en las clínicas de vacunación. Por otra parte, la pandemia por el coronavirus SARS-CoV-2 aún persiste y desconocemos la magnitud de su efecto en la vacunación contra VPH. Finalmente, cada uno de estos eventos nos hacen reflexionar como la legislación de política públicas pudo reducir potenciales barreras (cuadro 3) y que podrían informar a otros países en la adaptación de políticas públicas para la reducción de cánceres asociados al VPH.

Enfatizamos que la documentación de estos esfuerzos depende de registros robustos y confiables. Contar con un

CUADRO 2. Cambios en el requisito de vacunación contra el VPH para el ingreso a las escuela públicas y privadas de Puerto Rico desde su implementación en el 2018 de acuerdo con la Ley Núm. 25 de Inmunización de 25 de septiembre de 1983.

\begin{tabular}{ll}
\multicolumn{1}{l}{ Año Escolar } & Requisito por año escolar de acuerdo con la Ley 25 de 1983 \\
$2018-2019$ & 1 dosis de la vacuna contra el VPH en adolescentes de 11 a 12 años de edad y completar la serie según indicado (20) \\
$2019-2020$ & 1 dosis de la vacuna contra el VPH en adolescentes de 11 a 14 años de edad y completar la serie según indicado (33) \\
$2020-2021$ & 1 dosis de la vacuna contra el VPH en adolescentes de 11 a 16 años de edad y completar la serie según indicado (34) \\
\hline $\begin{array}{l}\text { Fuente: elaboración de los autores } \\
\text { VPH: virus de papiloma humano }\end{array}$ &
\end{tabular}


CUADRO 3. Reflexiones y recomendaciones sobre los eventos relacionados con la aprobación y aplicación de vacuna contra el VPH en Puerto Rico.

\begin{tabular}{|c|c|}
\hline Eventos & Reflexiones y recomendaciones \\
\hline $\begin{array}{l}\text { Aprobación de la vacuna contra el VPH y la legislación para su } \\
\text { cobertura médica }\end{array}$ & $\begin{array}{l}\text { Siendo PR un país donde sobre el } 40 \% \text { de las familias viven en un nivel de pobreza, esta legislación } \\
\text { significó un gran paso en la accesibilidad de la inmunización y la salud pública. }\end{array}$ \\
\hline Registro de Cáncer & $\begin{array}{l}\text { Comprender la función de los registros no solo para conocer la magnitud de los canceres que afectan } \\
\text { la población, sino que además al desarrollo de mejores servicios, acceso a tratamiento y evaluación de } \\
\text { efectividad de tratamiento son parte de su importancia. }\end{array}$ \\
\hline Registro de Inmunización & $\begin{array}{l}\text { Para evaluar campañas para la prevención de cáncer, a través de la vacunación se requiere que todos los } \\
\text { datos sean confiables y representativos de la población. }\end{array}$ \\
\hline Requisito Escolar & $\begin{array}{l}\text { La renuencia de muchos sectores ante este mandato se debe al hecho que VPH es una enfermedad de } \\
\text { transmisión sexual y no están viendo que es una vacuna para prevenir varios tipos de cáncer. Es necesario } \\
\text { una educación más enfocada en las dudas y preocupaciones que se tiene sobre la vacuna. }\end{array}$ \\
\hline Eventos climáticos o pandemia de COVID-19 & Se deben desarrollar protocolos para asegurar el servicio clínico y limitar problemas de salud pública \\
\hline
\end{tabular}

Fuente: elaboración de los autores

PR: Puerto Rico; VPH: virus de papiloma humano

registro de inmunización es vital para medir el estatus de vacunación por grupos de edad y estratos sociodemográficos. De esta forma, comprender el nivel de protección que cuenta la población sobre las enfermedades prevenibles por vacuna, y de ser necesario, a través de legislaciones garantiza un mayor nivel. Además, prospectivamente, se recomienda el desarrollo de registros para las lesiones causas por el VPH (i.e. verrugas, lesiones pre-cancerosas). Este sistema de monitoreo uniforme, sistemático y robusto ayudaría a documentar a corto plazo el impacto de este conjunto de estrategias implementadas para la prevención de VPH, principalmente, la vacuna. Por último, los gobiernos y las agencias de salud tanto de PR como de otros países deben considerar alinearse a las metas establecidas por la OMS para erradicar el cáncer cervical y reforzar los esfuerzos de campaña de educación, prevención, e investigación en su población.

Contribución de los Autores. DML, investigación, análisis formal, interpretación de datos, redacción del borrador, revisión y edición del manuscrito. VCL adquisición de fondos, supervisión, conceptualización, redacción del borrador original, investigación revisión y edición del manuscrito. VRF, análisis formal, redacción-preparación del borrador original, y revisión. CVO y GAM, elaboración de metodología, redacciónpreparación original, revisión y edición del manuscrito. RSA y ODM análisis formal, investigación, redacción, revisión y edición del manuscrito. APO adquisición de fondos, análisis formal, investigación, redacción, y revisión del manuscrito. LAC, PFR, IV, AR, IC y VOCES, escritura, revisión y edición. $\mathrm{PCH}$ adquisición de fondos, el análisis formal, investigación, redacción, revisión y edición.
Agradecimientos. Agradecemos al Programa de Control Comprensivo de Cáncer de Puerto Rico, afiliado al Centro Comprensivo de Cáncer de la Universidad de Puerto Rico (CCCUPR). Además, esta publicación fue apoyada por el CCC-UPR (corporación pública del Gobierno de Puerto Rico creada en virtud de la Ley 230 del 26 de agosto de 2004 según enmendada). El contenido es responsabilidad exclusiva de los autores y no necesariamente representa las opiniones oficiales del CCC-UPR.

Financiación. Este trabajo fue apoyado por National Institutes of Health; National Cancer Institute (subvención no. 1R01CA232743). Implementación de políticas de ingreso a la escuela para la vacunación contra el virus del papiloma humano en las escuelas. CVO fue apoyada por la Beca de Prevención del Cáncer del Instituto Nacional del Cáncer y Harvard T.H. Chan School of Public Health - Beca número 2T32CA057711-27 de los Institutos Nacionales de Salud. Sus contenidos son responsabilidad exclusiva de los autores y no representan necesariamente las opiniones oficiales del Instituto Nacional del Cáncer.

Conflicto de intereses. VCL recibió compensación de Merck and Co., Inc. por consultoría en junio de 2020. APO recibió compensación de Merck and Co., Inc en octubre de 2019 por consultoría. VOCES; Coalición de Inmunización y Promoción de la Salud de Puerto Rico ha recibido becas educativas de Merck and Co., Inc. desde 2013. Los otros autores no tienen conflicto financiero que revelar.

Declaración. Las opiniones expresadas en este manuscrito son responsabilidad del autor y no reflejan necesariamente los criterios ni la política de la $R P S P / P A J P H$ y /o de la OPS

\section{REFERENCIAS}

1. PanAmerican Health Organization. Cervical Cancer in Latin America and the Caribbean: Fact Sheet 2001 [Internet]. 2001 [consultado el 17 de agosto de 2021]. Disponible en: https://www.paho.org/ $\mathrm{hq} /$ dmdocuments/2011/Cervical Cancer in Latin America and the Caribbean.pdf

2. Torres-Cintrón CR, Alvarado-Ortiz M, Roman-Ruiz Y, Ortiz-Ortiz KJ, Zavala Zegarra DE, Tortolero-Luna G. Cancer in Puerto Rico,
2012-2016. Puerto Rico Cent Cancer Regist [Internet]. 2016;1-155. Disponible en: http://rcpr.org/Publicaciones/Reportes-Anuales

3. Registro Central de Cáncer de Puerto Rico. Base Legal-Nueva Ley del Registro Central de Cáncer de Puerto Rico, Ley Núm. 113 de 30 de julio de 2010 [Internet]. [consultado el 17 de agosto de 2021]. Disponible en: https://rcpr.org/Sobre-Nosotros/ Base-Legal 
4. Mirra AP. Registros de Cáncer en América Latina. Rev Bras Cancerol [Internet]. 1997 [consultado el 17 de agosto de 2021] ;43(1). Disponible en: https://rbc.inca.gov.br/site/arquivos/n_43/v01/ informe_completo.html

5. Parkin DM, Whelan SJ, Ferlay J, Teppo L, Thomas DB. Cancer Incidence in Five Continents. VIII. Lyon, France: International Agency for Research on Cancer; 2002. 1-831 p.

6. Ortiz AP, Ortiz-Ortiz KJ, Colón-López V, Tortolero-Luna G, Torres-Cintrón CR, Wu CF, et al. Incidence of Cervical Cancer in Puerto Rico, 2001-2017. JAMA Oncol. 2021;1;7(3):456-458

7. Viens LJ, Henley SJ, Watson M, Markowitz LE, Thomas CC, Thompson TD, et al. Human Papillomavirus-Associated Cancers - United States, 2008-2012. MMWR Morb Mortal Wkly Rep. 2016 Jul;65(26): $661-6$.

8. Ortiz-Ortiz KI, Ramos-Cartagena JM, Deshmukh AA, TorresCintrón CR, Colón-López V, Ortiz AP. Squamous Cell Carcinoma of the Anus Incidence, Mortality, and Survival Among the General Population and Persons Living With HIV in Puerto Rico, 2000-2016. JCO Glob Oncol. 2021;7:133-143.

9. Medina-Laabes DT, Suarez-Perez EL, Guiot HM, Muñoz C, Colón-López V, Tirado-Gómez M, et al. Human papillomavirus correlates with histologic anal high-grade squamous intraepithelial lesions in Hispanics with HIV. J Low Genit Tract Dis. 2018;22(4):320-5.

10. Colón-López V, Ortiz AP, Toro-Mejías L Del, Clatts M, DuránGuzmán G, Pérez N, et al. Prevalence and correlates of penile hpv infection in a clinic-based sample of hispanic males. P R Health Sci J. 2015;34(3):128-34.

11. Báez A, Almodóvar JI, Cantor A, Celestin F, Cruz-Cruz L, Fonseca S, et al. High frequency of HPV16-associated head and neck squamous cell carcinoma in the Puerto Rican population. Head Neck [Internet]. 2004 Sep 1 [consultado el 17 de agosto de 2021];26(9):778-84. Disponible en: https://onlinelibrary.wiley.com/doi/full/10.1002/ hed.20046

12. Pérez-Caraballo AM, Suarez E, Unger ER, Palefsky JM, Panicker G, Ortiz AP. Seroprevalence of Human Papillomavirus (HPV) Type 6, 11, 16, 18, by Anatomic Site of HPV Infection, in Women Aged 16-64 Years living in the Metropolitan Area of San Juan, Puerto Rico. P R Health Sci J [Internet]. 2018 Feb;37(1):26-31. Disponible en: http:/ / www.ncbi.nlm.nih.gov/pubmed/9547681

13. Markowitz LE., Dunne EF., Saraiya M, Chesson HW., Curtis CR, Gee J, et al. Human Papillomavirus Vaccination: Recommendations of the Advisory Committee on Immunization Practices (ACIP) [Internet]. Morbidity and Mortality Weekly Report (MMWR). 2014 [consultado el 25 de agosto de 2021]. Disponible en: https://www.cdc.gov/mmwr/preview/mmwrhtml/rr6305a1.htm\#tab1

14. LexJuris de Puerto Rico. Leyes por Años [Internet]. [consultado el 24 de septiembre de 2021]. Disponible en: https:/ / www.lexjuris.com/ lexleyes.htm

15. Red State Data Center de Puerto Rico. En pobreza el 50\% o más de la población en 36 Municipios de Puerto Rico | State Data Center [Internet]. Instituto de Estadísticas de Puerto Rico: Comunicado de Prensa. 2019 [consultado el 27 de septiembre de 2021]. Disponible en:: https://censo.estadisticas.pr/Comunicado-deprensa/2019-12-19t145558

16. Petrosky E, Bocchini J, Hariri S, Chesson H, Curtis R, Saraiya M, et al. Use of 9-Valent Human Papillomavirus (HPV) Vaccine: Updated HPV Vaccination RecommendationsUse of 9-Valent Human Papillomavirus (HPV) Vaccine: Updated HPV Vaccination Recommendations of the Advisory Committee on Immunization Practices. MMWR Morb Mortal Wkly Rep. 2015;27;64(11):300-4.

17. Meites E, Kempe A, Markowitz LE. Use of a 2-Dose Schedule for Human Papillomavirus Vaccination - Updated Recommendations of the Advisory Committee on Immunization Practices. MMWR Morb Mortal Wkly Rep [Internet]. 2016 Dec 16;65(49):1405-8. Disponible en: http://www.cdc.gov/mmwr/volumes/65/wr/ mm6549a5.htm

18. National HPV Roundtable. Milestones [Internet]. [consultado el 18 de agosto de 2021]. Disponible en: https://hpvroundtable.org/ milestones /

19. Puerto Rico Cancer Control Coalition, Puerto Rico Comprehensive Control Program. Puerto Rico Comprehensive Cancer Control Plan
2015-2020.[Internet] San Juan; 2014; [consultado el 18 de agosto de 2021]. Disponible en: https://www.iccp-portal.org/plans/ puerto-rico-comprehensive-cancer-control-plan-2015-2020

20. Vázquez-Otero $C$, Daley EM, Vamos CA, Romero-Daza N, Beckstead J, Martinez Tyson D. The Intersection of Problems, Policy, and Politics: The Adoption of an HPV Vaccine School-Entry Requirement in Puerto Rico. Qual Health Res [Internet] 2021;31(5):859-70. Disponible en: https://pubmed.ncbi.nlm.nih. gov/33733935/

21. Rivera Arguinzoni A. "Voces de Rhaiza" para concienciar sobre el virus del papiloma humano - El Nuevo Día. El Nuevo Día [Internet]. 2015 [consultado el 18 de agosto de 2021]; Disponible en: https://www.elnuevodia.com/noticias/locales/notas/ voces-de-rhaiza-para-concienciar-sobre-el-virus-del-papilomahumano/

22. Las Voces de Rhaiza Vélez Plumey. Las Voces de Rhaiza Vélez Plumey - Cáncer Cervical [Internet]. [consultado el 24 de septiembre de 2021]. Disponible en: https://www.facebook.com/ lasvocesderhaiza/

23. Asamblea Legislativa de Puerto Rico. Ley de las Inmunizaciones Compulsorias a los Niños Pre-escolares y Estudiantes en el Estado Libre Asociado de Puerto Rico [Ley 25-1983] [Internet]. Biblioteca Virtual del Gobierno de Puerto Rico. 1983 [consultado el 19 de agosto de 2021]. p. 1-6. Disponible en: https:/ / bvirtualogp.pr.gov / ogp/Bvirtual/leyesreferencia/PDF/25-1983.pdf

24. Luna-Pinto SC, Rivera A, Cardona I, Rijo C, Alvarez V, Rodriguez $\mathrm{J}$, et al. Restoring Immunization Services Provided by the Vaccines for Children Program in Puerto Rico After Hurricanes Irma and Maria, 2017-2019. J Public Heal Manag Pract [Internet]. 2020 Aug 6;27(6):E228-35. Disponible en: https:/ /journals.lww.com/10.1097/ PHH.0000000000001193

25. World Health Organization. Ten threats to global health in 2019 [Internet]. WHO- Spotlight. [consultado el 19 de agosto de 2021] Disponible en: https://www.who.int/news-room/spotlight/ ten-threats-to-global-health-in-2019

26. Medina Laabes DT, Rivera Encarnacion M, Soto-Abreu R, DíazMiranda O, Rivera-Figueroa V, Arroyo-Morales GO, Suárez-Perez, E, Fernández ME, Ortíz A, Hull P. Colón-López V. Abstract: Understanding HesitanT Parents' View Toward the Newly Enacted HPV Vaccine School Entry Policy in Puerto Rico. Poster presentado en: 33a International Papillomavirus Conference; 2020 Jul 20-24; Barcelona, España.

27. Rivas G, Soto-Abreu R, Arroyo-Morales GO, Medina-Laabes DT, Díaz-Miranda OL, Vázquez-Otero C, Colón-López V. Understanding Views Against the HPV School-Entry Requirement in Puerto Rico. Poster presentando en:Cancer Prevention and Control (CAPAC) Research Training Program Conference; 2021 Ago 05. San Juan, Puerto Rico:

28. Adhanom Ghebreyesus T. WHO Director-General's opening remarks at the media briefing on COVID-19 - 11 Marzo 2020 [Internet]. World Health Organization. 2020 [consultado el 13 de enero de 2021]. Disponible en: https://www.who.int/director-general/ speeches/detail/who-director-general-s-opening-remarks-at-themedia-briefing-on-covid-19---11-march-2020

29. Gilkey MB, Bednarczyk RA, Gerend MA, Kornides ML, Perkins RB, Saslow D, et al. Getting Human Papillomavirus Vaccination Back on Track: Protecting Our National Investment in Human Papillomavirus Vaccination in the COVID-19 Era. J Adolesc Health 2020;67(5):633-634

30. Colón-López V, Vázquez-Otero C, Rivera-Figueroa V, ArroyoMorales GO, Medina-Laabes DT, Soto-Abreu R, et al. HPV Vaccine School Entry Requirement in Puerto Rico: Historical Context, Challenges, and Opportunities. Prev Chronic Dis. 2021;18:E77

31. World Health Organization. Launch of the Global Strategy to Accelerate the Elimination of Cervical Cancer [Internet]. 2020 [[consultado el 26 de agosto de 2021]. Disponible en: https:/ / www.who. int/news-room/events/detail/2020/11/17/default-calendar/ launch-of-the-global-strategy-to-accelerate-the-elimination-ofcervical-cancer

32. Sistema Único de Trámite Legislativo. Registro de Medida: PC1576 [Internet]. Oficina de Servicios Legislativos . 2018 [consultado el 7 de abril de 2021]. Disponible en: https://sutra.oslpr.org/osl/ esutra/MedidaReg.aspx?rid=124048 
33. Departamento deEducación de Puerto Rico. Salud y Educación anuncian requisitos mínimos de vacunación y salud oral para el próximo año escolar-Departamento de Educación [Internet]. DEPR-Noticias. 2019 [consultado el 20 de agosto de 2021]. Disponible en:https: / /de.pr. gov/noticias/salud-y-educacion-anuncian-requisitos-minimosde-vacunacion-y-salud-oral-para-el-proximo-ano-escolar /

34. Departamento de Educación de Puerto Rico. Departamento de Salud orienta sobre vacunas requeridas a estudiantes para el nuevo semestre escolar [Internet]. DEPR-Schools. 2020 [consultado el 20 de agosto de 2021]. Disponible en: https://de.pr.gov/schools/ departamento-de-salud-orienta-sobre-vacunas-requeridas-a-estu diantes-para-el-nuevo-semestre-escolar/

Manuscrito recibido el 9 de abril del 2021. Aceptado para su publicación, tras revisión, el 7 de octubre del 2021.

\section{Efforts towards the consolidation of public policies for the prevention of HPV-associated cancers in Puerto Rico}

ABSTRACT The purpose of this special report is to describe chronologically the events that contributed to the development and approval of legislation and subsequent implementation of a school vaccination mandate in order to prevent HPV and HPV-associated cancers in Puerto Rico (PR). Starting in 2010, PR initiated publicpolicy approvals aimed at improving cancer registries and HPV vaccine coverage through health insurance for adolescents aged 11 to 18 years. In 2014, scientific and community efforts succeeded in documenting the magnitude of morbidity caused by HPV and jointly developing HPV vaccine prevention and promotion strategies. In August 2018, PR became one of the first four territories of the United States of America to implement the HPV vaccine school entry requirement to decrease the incidence of HPV-associated cancers on the island. In 2019, it was enshrined in law that every immunization provider must submit immunization data to the Puerto Rico Immunization Registry. The case of PR demonstrates that public policy-making alongside collaboration between academic, scientific, and community coalitions can achieve population change and measurable outcomes aimed at HPV prevention. Countries with a similar public health problem could adopt efforts similar to those presented herein and align them with the World Health Organization goal of eradicating cervical cancer by 2030 .

Keywords Papillomavirus vaccines; papillomavirus infections; disease prevention; health policy; Puerto Rico. 


\section{Esforços realizados em Porto Rico para a consolidação de políticas públicas de prevenção de cânceres associados ao HPV}

RESUMO O propósito deste relatório especial é descrever cronologicamente os eventos que contribuíram para o desenvolvimento e a aprovação de legislação, e a implementação da exigência escolar de vacinação em Porto Rico (PR), a fim de prevenir o HPV e os cânceres associados a ele. A partir de 2010, PR iniciou as aprovações de políticas públicas com o objetivo de aprimorar o registro dos casos de câncer e a cobertura vacinal contra o HPV, por meio de planos de saúde, em adolescentes de 11 a 18 anos. Em 2014, esforços científicos e comunitários permitiram documentar a magnitude das doenças causadas pelo HPV e elaborar conjuntamente estratégias de prevenção e promoção da vacina contra o HPV. Em agosto de 2018, PR foi um dos primeiros quatro territórios dos Estados Unidos da América a implementar a vacina contra o HPV como exigência escolar, a fim de diminuir a incidência de cânceres associados ao HPV na ilha. Em 2019 ficou garantido por lei que todos os vacinadores devem enviar informações ao Registro de Imunização. O caso de PR demonstra que o desenvolvimento de políticas públicas, em conjunto com parcerias entre coalizões acadêmicas, científicas e comunitárias, alcança mudanças populacionais e resultados mensuráveis dirigidos à prevenção do HPV. Países com uma problemática de saúde pública similar poderiam adotar esforços semelhantes aos apresentados e alinhá-los ao objetivo da Organização Mundial da Saúde: a erradicação do câncer cervical até 2030.

Palavras-chave Vacinas contra papillomavirus; infecções por papillomavirus; prevenção de doenças; política de saúde; Porto Rico. 